\title{
Phosphatidylinositol transfer proteins: From closed for transport to open for exchange
}

\author{
Karel W.A. Wirtz*, Arie Schouten, Piet Gros \\ Bijvoet Center for Biomolecular Research, Department of Lipid Biochemistry, Department of Crystal and Structural \\ Chemistry, Utrecht University, Utrecht, The Netherlands
}

\section{Introduction}

Since their purification from bovine brain phosphatidylinositol transfer proteins (PI-TP) have been subject to extensive studies mainly prompted by the hypothesis that these proteins play a key role in intracellular phosphatidylinositol (PI) transport and as a consequence, in PI-dependent signalling pathways (Helmkamp et al., 1974; Wirtz, 1997). To date, two isoforms of PI-TP have been identified: PI-TP $\alpha$ (270 amino acids) and PI-TP $\beta$ (271 amino acids). Either isoform $\left(\mathrm{M}_{\mathrm{r}} 32 \mathrm{kDa}\right)$ is highly conserved ( $>98 \%$ sequence identity) among mammalian species. Moreover, both proteins are highly homologous with a sequence identity of $77 \%$ (similarity of $94 \%$ ) yet appear to have distinctly different cellular functions. In agreement with this the gene of human PI-TP $\alpha$ is localized to chromosome 17q13 and of PI-TP $\beta$ to chromosome 22q12 (Ocaka et al., 2005). Both isoforms act as carriers of PI and phosphatidylcholine (PC) between membranes in vitro, yet PI-TP $\beta$ can also transfer sphingomyelin (SPM) (Van Paridon et al., 1987b; De Vries et al., 1995). At this point it is still questionable whether these proteins actually transfer these phospholipids between membranes within cells or whether they interact directly with enzymes like PI 4-kinases and PI-specific phospholipase $\mathrm{A}_{2}$ thereby providing these enzymes with substrates (Cunningham et al., 1995; Snoek et al., 1999). In analogy with observations in yeast it has also been proposed that by binding either PI, PC or SPM, PI-TPs act as regulators of phospholipid metabolism at distinct sites in the cell thereby fulfilling the roles of biosensors (McGee

\footnotetext{
*Corresponding author.

E-mail address: k.w.a.wirtz@chem.uu.nl (K.W.A. Wirtz).
} 
et al., 1994; Bankaitis, 2002). Although much remains to be elucidated about their physiological role an observation to be accounted for is that PI-TP $\alpha$ localizes predominantly to the nucleus and cytoplasm whilst PI-TP $\beta$ is mainly associated with the Golgi complex (De Vries et al., 1995, 1996). In line with being highly conserved in evolution both isomers are essential in cell function. So it was shown that murine embryonic stem (ES) cells deficient in PI-TP $\alpha$ developed normally during embryogenesis, yet the pups died early after birth stemming from complicated organ failure (Alb et al., 2003). On the other hand, PI-TP $\beta$ gene ablation failed to give viable ES cells (Alb et al., 2002). Very interestingly, a mutation in intron 4 of the PI-TP $\alpha$ gene was identified in the vibrator mouse (Hamilton et al., 1997). In these mice when homozygous in the vibrator $(v b)$ allele brain PI-TP $\alpha$ levels were five-fold reduced which was accompanied by progressive action tremor, degeneration of neurons in brain and spinal cord and early juvenile death.

\section{PI-TP domain-containing proteins}

Apart from PI-TP $\alpha$ and PI-TP $\beta$, mammalian tissues contain another soluble PItransferring protein $\operatorname{rdgB} \beta$ (332 amino acids) carrying a short carboxy-terminal domain and an amino-terminal PI-TP-like domain that is $41 \%$ identical to PI-TP $\alpha$ (Fullwood et al., 1999). This protein was identified by homology to the retinal degeneration protein (type B) in Drosophila, denoted as $\mathrm{Dm} \operatorname{rdgB} . \operatorname{RdgB} \beta$ is ubiquitously expressed in human tissues and diffusely present throughout the cytoplasm; the cellular functions are as yet unknown. Mammalian homologues of $\mathrm{Dm} \mathrm{RdgB}$ are rdgB1 (Nir2) (1244 amino acids) and rdgB2 (Nir3) (1349 amino acids) both of which proteins carry an amino-terminal PI-TP domain, a 15 -aa $\mathrm{Ca}^{2+}$-binding domain, a trans-membrane domain, a 180-aa conserved region containing the metal-binding motif DDHD and a highly conserved 300-aa carboxy-terminal segment containing the tyrosine kinase Pyk2-binding domain (Lev et al., 1999). The acronym Nir (N-terminal domain-Interacting Receptor) refers to the interaction of these proteins with the aminoterminal domain of tyrosine kinase Pyk2. The PI-TP domains of Nir2 and Nir3 are 45\% identical to PI-TP $\alpha, 72 \%$ identical with each other and $65 \%$ identical with the Drosophila homologue. Ablation of the mouse Nir2 gene resulted in early embryonic death whereas ablation of the Nir3 gene failed to produce a distinct phenotype (Lu et al., 2001). The PITP domain of Nir2 is involved in the production of PI(4)P supposedly by presenting PI to type III PI 4-kinase (Aikawa et al., 1999). Since Nir2 and PI 4-kinase are associated with the Golgi it was inferred that PI(4)P synthesis occurs in the Golgi membranes. By promoting $\mathrm{PI}(4) \mathrm{P}$ and $\mathrm{PIP}_{2}$ synthesis Nir2 plays an important role in membrane trafficking and cytoskeletal remodeling (Lev, 2004). Recently, Nir2 was found to play an essential role in maintaining a diacylglycerol (DAG) pool in the Golgi apparatus critical for controlling protein transport from the trans-Golgi network to the plasma membrane (Litvak et al., 2005). As previously shown for the yeast PI-TP $\beta$ (Sec14p) Nir2 maintains the Golgi DAG pool by regulating its consumption via the CDP-choline pathway (Bankaitis, 2002). Recently the genomic organization of both soluble and membrane-associated Drosophila and human PI-TP-domain containing genes was determined (Ocaka et al., 2005). Phylogenetic analysis indicated that the gene lineage of PI-TP $\alpha$ and PI-TP $\beta$ and the membrane-associated Dm RdgB, Nir2 and Nir3 arose at a very early stage in human evolution. 


\section{$3 D$-structure and phospholipid specificity}

\section{$P I-T P \alpha$}

A main feature of PI-TP $\alpha$ is its dual specificity expressing a 16-fold higher affinity for PI than for PC (van Paridon et al., 1987b). Recently, the structures of rat PI-TP $\alpha$ carrying a PC molecule and of human PI-TP $\alpha$ carrying a PI molecule were elucidated (Yoder et al., 2001; Tilley et al., 2004) (Fig. 1A). Both proteins give the same fold indicating that the structure is not affected by the kind of phospholipid bound. Either phospholipid is buried within the protein by occupying a lipid-binding cavity that allows the polar head group to interact with amino acid residues located at the far end of the cavity away from the opening through which the lipid has access to the membrane interface. The recognition sites for the phosphorylcholine and phosphorylinositol head group have in common those residues that make contact with the phosphate moiety, i.e. Gln22 and Thr114 (both hydrogen bonded via a water molecule), Lys195 to one phosphate-oxygen and Thr97 to the other phosphate oxygen (Fig. 2A). The residues that interact with the choline moiety are Glu86, Asn90 and Cys95 whereas the inositol moiety interacts with Glu86, Asn90, Thr59 and Lys61 through hydrogen bonds. Given the hydrogen bonding with the inositol moiety we may presume that PI is more tightly bound to the protein than PC. This was confirmed by electrospray time-of-flight mass spectrometry analysis of the PI-TP $\alpha$-lipid complexes (De Brouwer et al., 2002). Both structures indicate that the sn-1 and sn-2 fatty acyl chain of either ligand occupy distinct sites with the methyl ends being close to the opening of the lipid-binding cavity which is shielded from the outside by the lipid exchange loop (helix B) and the C-terminal end (residues 261-271) as part of helix G (Fig. 1A). By time-resolved fluorescence spectroscopy we have shown that the parinaroyl-chain attached at the sn-2 position of either PI or PC was completely immobilized in the lipid-binding cavity (van Paridon et al., 1987a). Given that rotational correlation times were very similar

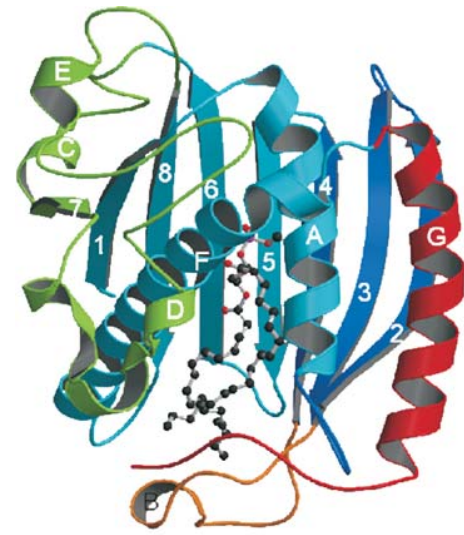

(A)

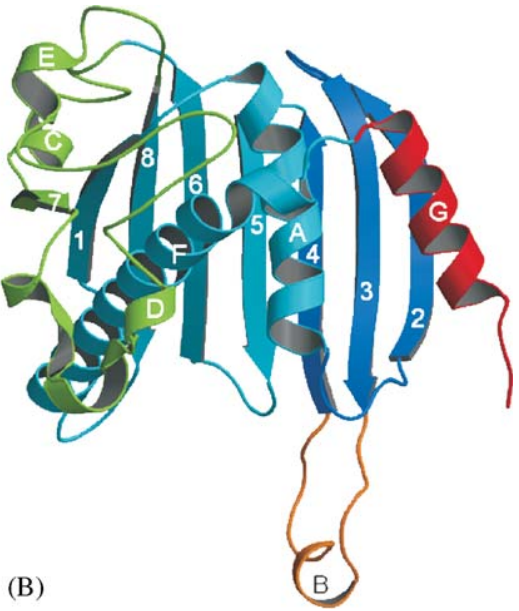

(B)

Fig. 1. A ribbon diagram of the closed (A) and open (B) conformation of PI-TP $\alpha$ from rat and mouse, respectively. The structures contain four functional regions, i.e. the lipid binding core (blue) with the flexible part of the $\beta$-strand (dark blue), the regulatory loop (green), the C-terminal region (red) and the lipid exchange loop (orange). The lipid molecule bound to the closed form is PC. 


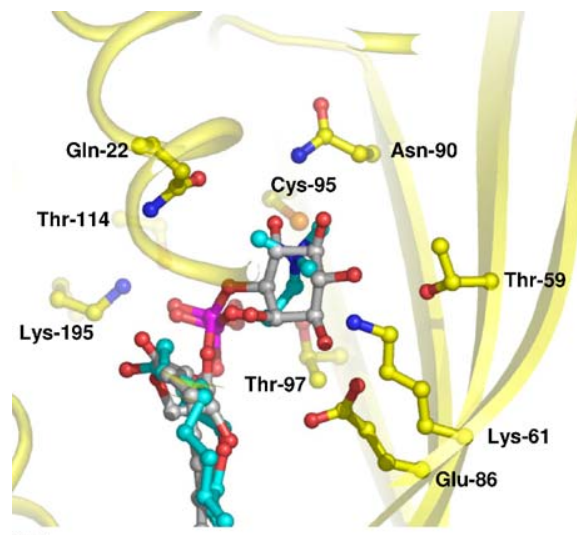

(A)

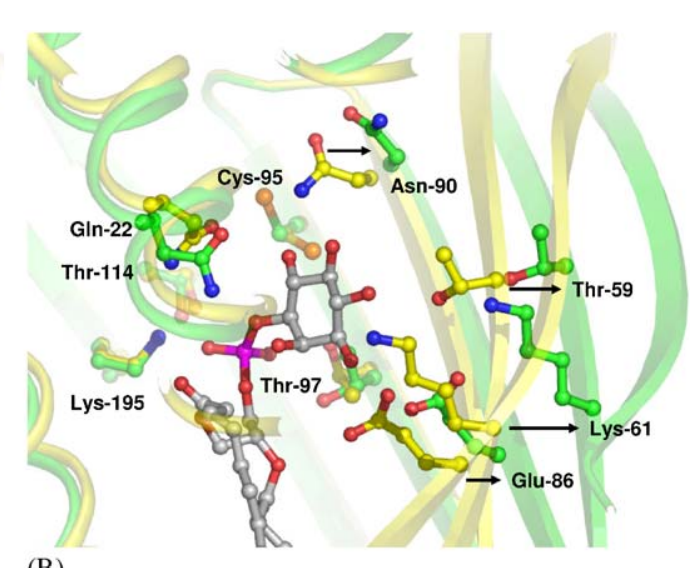

(B)

Fig. 2. Binding sites for PI and PC in the closed (A) and open (B) form of PITP $\alpha$. Shown are ribbons of closed (yellow) and open (green) PITP around the polar head-group binding site. Residues involved in the interactions, PI (grey) and PC (blue) are shown in ball-and-stick model. PI in the open form is depicted in the same position as in the closed form. Arrows in Figure indicate the conformational shift corresponding to the opening of the lipidbinding site.

we inferred that as now shown by X-ray analysis the sn-2 fatty acyl chains of both phospholipids were accommodated in the same hydrophobic binding site of the protein.

The detailed knowledge about how PC and PI are bound to the protein raises the question what happens at the membrane interface when upon interaction PI-TP $\alpha$ releases its bound PC in exchange for PI from the outer monolayer and vice versa thereby expressing a distinct preference for PI over PC. Recently we have elucidated the structure of PI-TP $\alpha$ devoid of a bound phospholipid molecule (Schouten et al., 2002) (Fig. 1B). This structure reveals an open conformation by which the lipid exchange loop (helix B) has moved outward and the $\mathrm{C}$-terminal helix $\mathrm{G}$ is dislodged to the extent that the lipid-binding cavity is now accessible from the outside. Another striking feature is that the outward movement of the lid is accompanied by a widening of the lipid-binding cavity creating a large opening $\left(350 \AA^{2}\right.$ ) at the site of the lipid exchange loop. By flattening of the $\beta$-sheet (strands $2-4)$ a small opening $\left(75 \AA^{2}\right)$ is formed at the opposite side of the protein resulting in the formation of a channel. The large opening exposes a hydrophobic surface whereas the small opening is of hydrophilic nature and close to the site for lipid head group binding. We assume that this open conformation reflects the membrane-associated state of $\mathrm{PI}-\mathrm{TP} \alpha$ with the large opening exposed to the membrane interface.

As a consequence of the formation of the lipid binding channel, the binding site for the phosphorylinositol head group is shifted towards the small channel opening with Thr59 and Lys61 ( $\beta$-strand 3 ) being displaced $4-4.5 \AA$ (C $\alpha$ to $\mathrm{C} \alpha$ distance) and Glu86 and Asn90 ( $\beta$-strand 4) $1-1.5 \AA$ relative to the positions in the closed conformation (Fig. 2B). For comparison, the modest displacement of Glu86 and Asn90 and the fixed position of Cys95 indicate that the binding site for the phosphorylcholine head group remains in place. In addition, the residues of the phosphate-binding site (i.e. Gln22, Thr97, Thr144, Lys195) are not displaced in the open conformation (Fig. 2B). On the other hand, provided the phosphorylinositol moiety in the open conformation remains in its binding site, the phosphate of PI is shifted about $4 \AA$ away from the phosphate of PC in PC-PITP $\alpha$ towards 
the channel opening limiting hydrogen bonding to Gln22 and Thr97. Under these conditions water molecules entering through the small channel opening may interfere with the hydrogen-bonding network thereby destabilizing PI in its lipid binding site and facilitating its release into the membrane interface. On the other hand, we propose that the separate binding sites for each moiety in the open conformation explain as to why the protein transfers PI at a higher rate than PC. Upon dissociation from the interface the protein returns to its closed conformation in the process of which water molecules must be again extruded from the lipid-binding channel.

What is the evidence for separate phosphorylcholine and phosphorylinositol binding sites in the open conformation? For one thing, PI-TP $\alpha$ does not transfer other phospholipids like phosphatidic acid, phosphatidylethanolamine and phosphatidylserine, yet it does transfer phosphatidylglycerol (PG) (Somerharju et al., 1983). This most likely indicates that the phosphorylglycerol polar head moiety can form the necessary hydrogen bonds when exposed to the lipid-binding cavity. Furthermore, by mutating the amino acid residues constituting the hydrogen bonding partners in the phosphorylinositol-binding site (i.e. Glu86, Asn90, Thr59) it was observed that the mutant PI-TP $\alpha$ 's (K61A, N90F, N90L, T59E) do not or barely bind and transfer PI whereas binding and transfer of PC is unaffected (Alb et al., 1995; Tilley et al., 2004). This strongly suggests that the phosphorylcholine-binding site of these mutant proteins is functioning normally. Other mutant proteins (T59A, T59S, E86A) also showed a diminished binding and transfer of PI, yet little effect on the binding and transfer of PC was observed.

Previous studies on phospholipid monolayers spread at the air-water interface indicated that PI-TP $\alpha$ injected into the subphase rapidly exchanged its bound ligand for a molecule from the monolayer (Demel et al., 1977). We now presume that upon interacting with the lipid interface the protein exposes the hydrophobic cavity of the open conformation thereby allowing phospholipids to freely move between monolayer and lipid binding site. Given that we are dealing with a very dynamic situation where the protein goes through a 'closed-open-closed cycle' the interaction with the membrane cannot be too strong. Hence for the cycle to function there must be a subtle balance between hydrophobicity and hydrophilicity of the residues exposed at the interface (Fig. 3). We propose that the residues indicated (i.e. K68, R74, F72, Y103, K156, W203, W204, K209) effectively contribute to this balance and, hence, are important for PI-TP $\alpha$ to effectively shuttle phospholipids between membrane interfaces. A common feature of these residues is that except for Phe72 the side-chains have an amphipathic character making them well suited to interact with a membrane interface. Selection of these residues was based on their being conserved between PI-TP-domain containing proteins (see below). In support of this propose role, the mutant (WW 203/204 AA) does not transfer PI and PC whereas the mutant (Y103A) retained about $50 \%$ of the activity (Tilley et al., 2004). On the other hand, the mutant (F72A) was fully active suggesting that the replacement of Phe with Ala does not significantly disturb the hydrophobic-hydrophilic balance. The relatively high propensity of positively charged residues would also explain why PI-TP $\alpha$ is strongly associated with negatively charged membrane interfaces, thereby inhibiting the transfer activity (Somerharju et al., 1983).

As yet, it is not at all clear how PI-TP $\alpha$ interacts with PI 4-kinase for PI to become available for phosphorylation or with phospholipase $A_{2}$ for PI to become hydrolyzed (Cunningham et al., 1995; Snoek et al., 1999). In the open structure of PI-TP $\alpha$ the hydroxyl groups at positions 3,4 and 5 of the phosphorylinositol moiety are located in front of the 


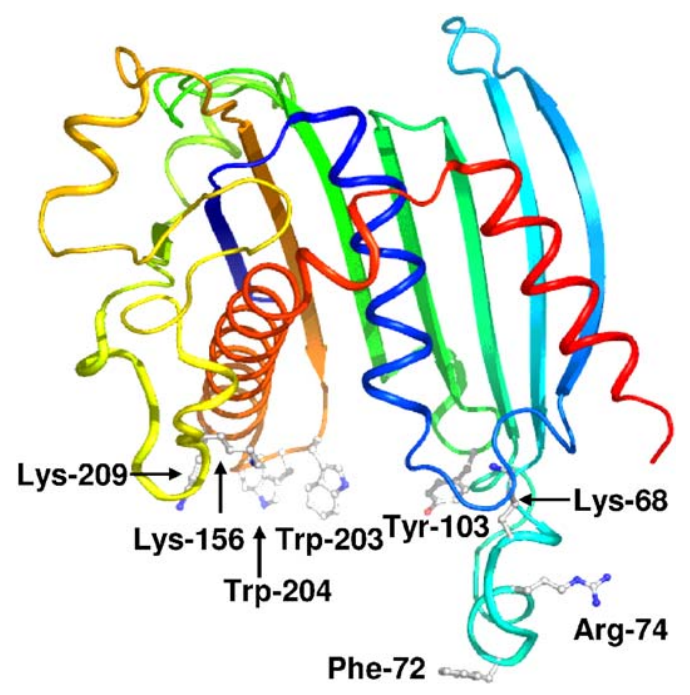

Fig. 3. Position of the amino acid residues involved in the association of PI-TP $\alpha$ with the membrane interface. The residues indicated are highly conserved in PI-TP $\beta$ and in the PI-TP domains of human $\operatorname{rdgB} \beta, \operatorname{Nir} 2$ and Nir3 and of Drosophila rdgB (Fullwood et al., 1999)

hydrophilic hole of the lipid-binding channel (Schouten et al., 2002). Given the size of the small opening it is unlikely that PI-kinases can directly phosphorylate PI. Hence it is fair to assume that these enzymes act on PI no longer bound to PI-TP $\alpha$ and that possibly ancillary proteins are involved.

\section{PI-TP $\beta$ and PI-TP domain-containing proteins}

Given the very high sequence homology it is most likely that the structure of PI-TP $\beta$ is very similar to that of PI-TP $\alpha$ (Snoek et al., 2004). Of the 24 residues that are functionally different most changes are found in the regulatory loop mainly consisting of helices $\mathrm{C}-\mathrm{E}$ and in the lid-forming C-terminal helix $G$ (Fig. 1). Only two amino acid residues are different in the lipid-binding region: Ile84 and Phe225 in PI-TP $\alpha$ are replaced by Phe and Leu in PI-TP $\beta$, respectively. However, the residues forming the polar head-binding site (i.e. Gln22, Thr59, Lys61, Glu86, Asn90, Thr97, Thr114, Lys195) are completely conserved, not only in PI-TP $\alpha$ and PI-TP $\beta$, but also in the soluble $\operatorname{rdgB} \beta$ from Drosophila and human and in the PI-TP domain of the membrane-associated Dm RdgB, rdgB1 (Nir2) and rdgB2 (Nir3) (Fullwood et al., 1999). In addition, the residues proposed to be involved in the association of PI-TP $\alpha$ with the membrane interface (i.e. residues of the lipid binding core and the lipid exchange loop, see Fig. 3) are conserved for the most part in the membraneassociated Dm RdgB, Nir2 and Nir3 (Fullwood et al., 1999; Schouten et al., 2002). This high level of identity agrees with the recombinants of the PI-TP domains being active in the PI-transfer assay. At this stage we do not know whether the PI-TP domain being normally closely positioned to the membrane, goes through the motion of opening and closing whilst binding and transferring PI. Given that the PI-TP domain is crucial for the functioning of these membrane-associated proteins we may infer that these domains are involved in the delivery of PI to enzymes like PI 4-kinase (Cockcroft, 1999; Lev, 2004). 


\section{Protein kinase C-dependent phosphorylation}

\section{Phosphorylation of solvent-inaccessible Ser 166}

PI-TP $\alpha$ and PI-TP $\beta$ are substrates for protein kinase C (van Tiel et al., 2000, 2002). Phosphoamino acid analysis of in vitro ${ }^{32} \mathrm{P}$-labeled PI-TPs demonstrated that both isoforms were exclusively phosphorylated on a serine residue. Based on prediction analyses of the amino acid sequences PI-TP $\alpha$ has one serine residue as a putative PKC-dependent phosphorylation site (i.e. Ser-166) and PI-TP $\beta$ two serine residues (i.e. Ser-165 and Ser262). These serine residues were positively identified as the phosphorylation sites by using mutant PI-TP $\alpha$ (S166A) and mutant PI-TP $\beta$ s (S165A, S262A, S165A/S262A) as substrates for PKC. As for PI-TP $\beta$, Ser165 was identified as the minor and Ser262 as the major phosphorylation site (van Tiel et al., 2002). Also in PI-TP $\alpha$ Ser166 was phosphorylated to a very limited extent. Recently it was confirmed that PI-TP $\alpha$ is phosphorylated in vitro by PKC at Ser166, yet a minor phosphorylation of Thr59 was also observed (Morgan et al., 2004). These sites of phosphorylation are difficult to reconcile with the 3D-structures of holo- and apoPI-TP $\alpha$, which clearly indicate that Ser166 is solvent inaccessible being present in a small pocket between the 165-172 loop and the remainder of the protein whereas Thr59 is present in the lipid binding cavity. As shown in Fig. 4A, the $\gamma-\mathrm{OH}$ of Ser166 forms a strong hydrogen-bond (2.6 ) with Asp234 (O $\delta 1)$ of helix F. The configuration of the 165-172 loop is further stabilized by hydrogen bonds between Asp234 $(\mathrm{O} \delta 2)$ and the $167 \mathrm{NH}$ function and between Asp234 (CO, peptide bond) and the $\varepsilon$-amino group of Lys168 (2.7 $\AA$ ) and by a salt bridge between Glu17 and Arg171. This network is completely conserved between soluble and membrane-associated proteins. Given that phosphorylation does occur we must assume that a small fraction of PI-TP $\alpha$ can adopt a structure in which Ser166 is accessible to PKC. This structure may possibly be induced upon interaction of PI-TP $\alpha$ with the interface formed by the activators of PKC, i.e. phosphatidylserine and diglyceride. It has also been proposed that upon docking to the membrane PI-TP $\alpha$ undergoes structural changes more extensively than observed for the open conformation, including relaxation of the 165-172 loop such that Ser166 becomes

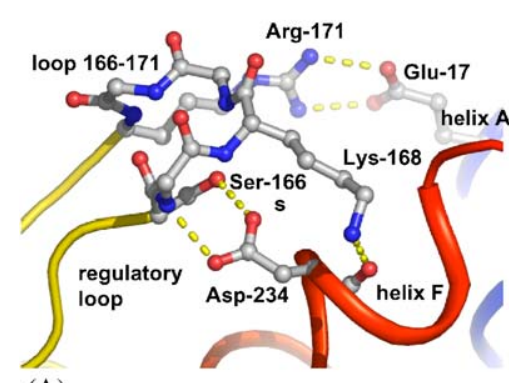

(A)

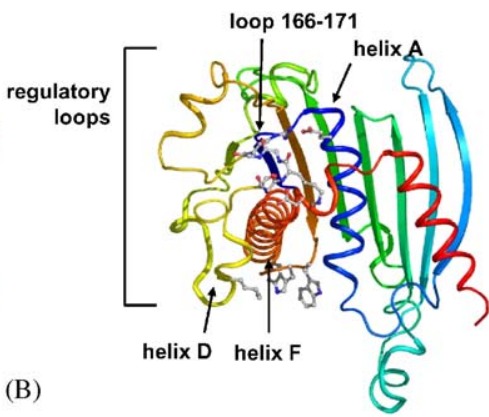

Fig. 4. The hydrogen-bonding network of loop 165-172 containing the PKC-dependent phosphorylation site Ser166. A, Ser-166 is solvent-inaccessible forming strong hydrogen bonds with Asp-234. The conformation of loop 165-172 is further stabilized by hydrogen bonds between Lys-168 and O-234 and by a salt bridge between Arg-171 and Glu-17. The loop 165-172 indicates the fold of the main chain and the orientation of the side chains of Ser166, Lys168 and Arg171. B, Spatial relationship between loop 165-172 and the regulatory loop including helix D and helix F. Disruption of the hydrogen bond between Ser-166 and Asp-234 may cause a reorientation of Lys-156 in helix D and Trp-203 and Trp204 in helix F (see Fig. 3) thereby rendering the protein inactive. 
solvent accessible (Morgan et al., 2004). More specifically, Snoek et al. (2004) have proposed that interaction of PI-TP $\alpha$ with the membrane may induce a slight reorientation of helix F. Since Ser166 is located at a distance of about $6 \AA$ above the axis of helix F, this reorientation could trigger a slight conformational change leading to solvent exposure of the $\gamma$-OH function of Ser166. Based on a kinetic analysis it was observed that the PCcontaining form of PI-TP $\alpha$ was phosphorylated at a faster rate than the PI-containing form (van Tiel et al., 2000). In line with this, the mutant PI-TP $\alpha$ (T59A), which is unable to bind PI, was a better substrate for PKC than the wtPI-TP $\alpha$ (Morgan et al., 2004). These observations suggest that subtle changes in the polar head region of the lipid-binding cavity induce a shift of the PI-TP $\alpha$ structure towards the PKC-accessible form.

\section{Ser166 is a key residue in the regulatory loop}

Ser-166 is conserved in 36 out of $39 \mathrm{PI}-\mathrm{TP} \alpha$-related sequences identified in mammals, fish, amphibians, flies, soil amoebae, red photosynthetic alga, and parasites suggesting an important role of this residue for protein function (Morgan et al., 2004). This residue is part of the regulatory loop and approximately $15 \AA$ away from the membrane-association site (Fig. 4B). Despite this distance Ser166 is essential for phospholipid transfer activity as the mutant PI-TP $\alpha$ s (S166A, S166D, S166E) fail to transfer PI and PC between membranes (Van Tiel et al., 2000; Morgan et al., 2004). Since the phosphomimetic mutants (S166D, $\mathrm{S} 166 \mathrm{E})$ fail to express transfer activity we may assume that phosphorylated PI-TP $\alpha$ is inactive as well. This also holds for PI-TP $\beta$ where the mutant PI-TP $\beta$ (S165A) lacks transfer activity (Van Tiel et al., 2002). As can be seen from Fig. 4A the hydrogen-bond between Ser166 and Asp234 is absent in the mutant PI-TP $\alpha(\mathrm{S} 166 \mathrm{~A})$ whereas strong repulsions occur between the Asp166/Glu166 and Asp234 in the mutant PI-TP $\alpha$ (S166D, $\mathrm{S} 166 \mathrm{E})$. A similar repulsion will occur between phosphorylated Ser166 and Asp234 in wt $\mathrm{PI}-\mathrm{TP} \alpha$. Apparently, disruption of the hydrogen-bond by phosphorylation (or just mutation) of Ser166 locks PI-TP $\alpha$ in the conformation of an inactive state. This inactivation may be due to subtle changes in the orientation of helix $\mathrm{F}$ and/or the regulatory loop including helix D thereby affecting the interaction of Trp203, Trp204, Lys209 and Lys156 with the membrane interface (Fig. 4B). These amino acids belong to the highly conserved residues of the membrane-association site (see Fig. 3).

We do not yet know whether this phosphorylation occurs before or after release of the bound lipid into the membrane. Receptor-mediated activation of phospholipase $\mathrm{C}$ resulting in diglyceride production and subsequent $\mathrm{PKC}$ activation at the plasma membrane has been proposed to trigger the phosphorylation of PI-TP $\alpha$ (Van Tiel et al., 2000; Morgan et al., 2004). If so, then it appears that this phosphorylated form can relocalize from the plasma membrane to perinuclear Golgi structures (Van Tiel et al., 2000). In contrast to Myc-tagged wtPI-TP $\alpha$, Myc-tagged mutants (S166A, S166D) expressed in NIH3T3 cells did not relocalize to the Golgi structures upon PMA stimulation, strongly suggesting that phosphorylation of Ser166 is required for relocalization. At this stage it is not known how phosphorylation and relocalization affect the functioning of PI-TP $\alpha$ in the cell. In this connection it is to be noted that PI-TP $\beta$ is found to be predominantly associated with the Golgi membranes as a consequence of the constitutive phosphorylation of the major PKC- phosphorylation site Ser-262 (Van Tiel et al., 2002). In agreement with this, inhibition of in situ phosphorylation with the PKC inhibitor GF 109203X or expressing PI-TP $\beta$ (S262A) into NIH3T3 fibroblasts showed that under these conditions PI-TP $\beta$ is distributed throughout the cytoplasm. To investigate the 
role of Ser-262 in the functioning of PI-TP $\beta$ in the cell, wtPI-TP $\beta$ and PI-TP $\beta$ (S262A) were overexpressed in NIH3T3 fibroblast cells. In contrast to the wtPI-TP $\beta$ overexpressors, the $\mathrm{PI}-\mathrm{TP} \beta(\mathrm{S} 262 \mathrm{~A})$ overexpressors were unable to rapidly replenish sphingomyelin in the plasma membrane upon degradation by sphingomyelinase. This implies that the association with the Golgi body is a prerequisite for PI-TP $\beta$ to express its effect on sphingomyelin metabolism (van Tiel et al., 2002).

\section{Summary}

PI-transfer proteins and PI-TP-domain containing proteins (e.g. Drosophila and human retinal degeneration proteins, Dm $\mathrm{rdgB}$, Nir 2 and Nir3) play very important roles in cellular lipid metabolism. In agreement with their dual specificity, these proteins have fully conserved binding sites for the polar head groups of PI and PC. Whereas in the closed conformation the polar head binding sites coincide, the open conformation of PI-TP $\alpha$ strongly suggests that at the membrane interface the binding site for the phosphorylinositol head group is shifted relative to that of the phosphorylcholine head group. In addition, residues important for the interaction of these proteins with the membrane interface are highly conserved. To date, virtually all PI-transferring proteins have been identified to contain an essential Ser166 residue in the regulatory loop. From the 3D-structures it can be derived that this residue is solvent-inaccessible and kept in place by a highly conserved hydrogen-bonding network. Disruption of the hydrogen bond between Ser166 and Asp234 by replacing the Ser166 residue inactivates the mutant PI-TPs (S166A, S166E, S166D). A model is presented how mutation or protein kinase $\mathrm{C}$-dependent phosphorylation of Ser166 may affect the interaction of PI-TP with the membrane interface. The limited PKCdependent phosphorylation observed implies that PI-TP may adopt a conformation allowing a reorientation of the $\gamma-\mathrm{OH}$ function of Ser166. It has been proposed that this reorientation may be induced by PI-TP interacting with the membrane interface.

\section{Acknowledgements}

The author (KWAW) is grateful to the Council for Chemical Sciences of the Netherlands Organization for Scientific Research (NWO-CW) for the continuous support through the years.

\section{References}

Aikawa Y, Kuraoka A, Kondo H, Kawabuchi M, Watanabe T. Involvement of PITPnm, a mammalian homologue of Drosophila rdgB, in phosphoinositide synthesis on Golgi membranes. J Biol Chem 1999;274: 20569-77.

Alb JG, Gedvilaite A, Cartee RT, Skinner HB, Bankaitis VA. Mutant rat phosphatidylinositol/phosphatidylcholine transfer proteins specifically defective in phosphatidylinositol transfer: implications for the regulation of phospholipid transfer activity. Proc Natl Acad Sci USA 1995;92:8826-30.

Alb JG, Phillips SE, Rostand K, Cui X, Pinxteren J, Cotlin L, Manning T, Guo S, York JD, Sontheimer H, Collawn JF, Bankaitis VA. Genetic ablation of phosphatidylinositol transfer protein function in murine embryonic stem cells. Mol Biol Cell 2002;13:739-54.

Alb JG, Cortese JD, Phillipis SE, Albin RL, Nagy TR, Hamilton BA, Bankaitis VA. Mice lacking phosphatidylinositol transfer protein-alpha exhibit spinocerebellar degeneration, intestinal and hepatic steatosis, and hypoglycemia. J Biol Chem 2003;278:33501-18.

Bankaitis VA. Slick recruitment to the Golgi. Science 2002;295:290-1. 
Cockcroft S. Mammalian phosphatidylinositol transfer proteins: emerging roles in signal transduction and vesicular traffic. Chem Phys Lipids 1999;98:23-33.

Cunningham E, Thomas GM, Ball A, Hiles I, Cockcroft S. Phosphatidylinositol transfer protein dictates the rate of inositol trisphosphate production by promoting the synthesis of PIP2. Curr Biol 1995;5:775-83.

De Brouwer APM, Versluis C, Westerman J, Roelofsen B, Heck AJR, Wirtz KWA. Determination of the stability of the noncovalent phospholipid transfer protein-lipid complex by electrospray time-of-flight mass spectrometry. Biochemistry 2002;41:8013-8.

De Vries KJ, Heinrichs AA, Cunningham E, Brunink F, Westerman J, Somerharju P J, Cockcroft S, Wirtz KWA, Snoek GT. An isoform of the phosphatidylinositol-transfer protein transfers sphingomyelin and is associated with the Golgi system. Biochem J 1995;310:643-9.

De Vries KJ, Westerman J, Bastiaens PI, Jovin TM, Wirtz KWA, Snoek GT. Fluorescently labeled phosphatidylinositol transfer protein isoforms (alpha and beta), microinjected into fetal bovine heart endothelial cells, are targeted to distinct intracellular sites. Exp Cell Res 1996;227:33-9.

Demel RA, Kalsbeek R, Wirtz KWA, Van Deenen LLM. The protein-mediated net transfer of phosphatidylinositol in model systems. Biochim Biophys Acta 1977;466:10-22.

Fullwood Y, dos Santos M, Hsuan JJ. Cloning and characterization of a novel human phosphatidylinositol transfer protein, rdgBbeta. J Biol Chem 1999;274:31553-8.

Hamilton BA, Smith DJ, Mueller KL, Kerrebrock AW, Bronson RT, van Berkel V, Daly MJ, Kruglyak L, Reeve MP, Nemhauser JL, Hawkins TL, Rubin EM, Lander ES. The vibrator mutation causes neurodegeneration via reduced expression of PITP alpha: positional complementation cloning and extragenic suppression. Neuron 1997;18:711-22.

Helmkamp GM, Harvey MS, Wirtz KWA, Van Deenen LLM. Phospholipid exchange between membranes. Purification of bovine brain proteins that preferentially catalyze the transfer of phosphatidylinositol. J Biol Chem 1974;249:6382-9.

Lev S. The role of the Nir/rdgB protein family in membrane trafficking and cytoskeleton remodeling. Exptl Cell Res 2004;297:1-10.

Lev S, Hernandez J, Martinez R, Chen A, Plowman G, Schlessinger J. Identification of a novel family of targets of PYK2 related to Drosophila retinal degeneration B (rdgB) protein. Mol Cell Biol 1999;19:2278-88.

Litvak V, Dahan N, Ramachandran S, Sabanay H, Lev S. Maintenance of the diacylglycerol level in the Golgi apparatus by the Nir2 protein is critical for Golgi secretory function. Nat Cell Biol 2005;7:225-34.

Lu C, Peng YW, Shang J, Pawlyk BS, Yu F, Li T. The mammalian retinal degeneration B2 gene is not required for photoreceptor function and survival. Neuroscience 2001;107:35-41.

McGee TP, Skinner HB, Whitters EA, Henry SA, Bankaitis VA. A phosphatidylinositol transfer protein controls the phosphatidylcholine content of yeast Golgi membranes. J Cell Biol 1994;124:273-87.

Morgan CP, Skippen A, Segui B, Ball A, Allen-Baume V, Larijani B, Murray-Rust J, McDonald N, Sapkota G, Morrice N, Cockcroft S. Phosphorylation of a distinct structural form of phosphatidylinositol transfer protein alpha at Ser166 by protein kinase C disrupts receptor-mediated phospholipase C signaling by inhibiting delivery of phosphatidylinositol to membranes. J Biol Chem 2004;279:47159-71.

Ocaka L, Spalluto C, Wilson DI, Hunt DM, Halford S. Chromosomal localization, genomic organization and evolution of genes encoding human phosphatidylinositol transfer protein membrane-associated (PITPNM) 1,2 and 3. Cytogenet Genome Res 2005;108:293-302.

Schouten A, Agianian B, Westerman J, Kroon J, Wirtz KWA, Gros P. Structure of apo-phosphatidylinositol transfer protein alpha provides insight into membrane association. Embo J 2002;21:2117-21.

Snoek GT, Berrie CP, Geijtenbeek TB, van der Helm HA, Cadee JA, Iurisci C, Corda D, Wirtz KWA. Overexpression of phosphatidylinositol transfer protein alpha in NIH3T3 cells activates a phospholipase A. J Biol Chem 1999;274:35393-9.

Snoek GT, Van Tiel CM, Egmond MR. Structure-function relationships of phosphatidylinositol transfer proteins: involvement of phosphorylation sites. Biochimie 2004;86:857-64.

Somerharju P, Van Paridon P, Wirtz KWA. Phosphatidylinositol transfer protein from bovine brain. Substrate specificity and membrane binding properties. Biochim Biophys Acta 1983;731:186-95.

Tilley SJ, Skippen A, Murray-Rust J, Swigart PM, Stewart A, Morgan CP, Cockcroft S, McDonald NQ. Structure-function analysis of human phosphatidylinositol transfer protein alpha bound to phosphatidylinositol. Structure 2004;12:317-26.

Van Paridon PA, Visser AJ, Wirtz KWA. Binding of phospholipids to the phosphatidylinositol transfer protein from bovine brain as studied by steady-state and time-resolved fluorescence spectroscopy. Biochim Biophys Acta 1987a;898:172-80. 
Van Paridon PA, Gadella TW, Somerharju PJ, Wirtz KWA. On the relationship between the dual specificity of the bovine brain phosphatidylinositol transfer protein and membrane phosphatidylinositol levels. Biochim Biophys Acta 1987b;903:68-77.

Van Tiel CM, Westerman J, Paasman M, Wirtz KWA, Snoek GT. The protein kinase C-dependent phosphorylation of serine 166 is controlled by the phospholipid species bound to the phosphatidylinositol transfer protein alpha. J Biol Chem 2000;275:21532-8.

Van Tiel CM, Westerman J, Paasman MA, Hoebens MM, Wirtz KWA, Snoek GT. The Golgi localization of phosphatidylinositol transfer protein beta requires the protein kinase C-dependent phosphorylation of serine 262 and is essential for maintaining plasma membrane sphingomyelin levels. J Biol Chem 2002;277:22447-52.

Wirtz KWA. Phospholipid transfer proteins revisited. Biochem J 1997;324:353-60.

Yoder MD, Thomas LM, Tremblay JM, Oliver RL, Yarbrough LR, Helmkamp GM. Structure of a multifunctional protein. Mammalian phosphatidylinositol transfer protein complexed with phosphatidylcholine. J Biol Chem 2001;276:9246-52. 